

\section{Okun's Law and Long Term Co-Integration Analysis for OECD Countries (1987-2012)}

\section{Bilal Kargı}

\section{Introduction}

A. Okun (1962), to explain relation between unemployment and growth, stated that an increase in employment should induce an increase in the level of GDP and unemployment will cause GDP to decrease. This reverse relation between GDP and unemployment is defined as Okun's Law (Mankiw, 2009: 260-263; Plosser and Schwert, 1979; Lang and De Peretti, 2009; Teck, 2012). There are some special cases for Okun's Law. Increase of working hours of current employees may create increase in GDP, if it does not cause increase in unemployment (Knotek 2007; Levine, 2013).

While average unemployment rate of the period 1970 to 2012 for OECD countries (OECD.StatExtract) was $5.586 \%$, it is found to be $8.430 \%$ when calculated for the period 2000 to 2012. GDP is increased $2.747 \%$ for the period 1970 to 2012, but for the period 2000 to 2012, it increased $1.871 \%$. Both data are given in the Graph 1.

GDP graph starting from 1970 also shows 1974, 1981 and 2008 crisis. Most influential crisis is the 2008 crisis in the way of its affecting unemployment mostly. Especially at the end of 80 's, unemployment rates started to increase while GDP continued its characteristic movements. Reason of this can be shown as; developments in the use of technology and directing to non-OECD countries with cheap facilities and labor force.

Graph 1: $\quad$ GDP growth rate (Red) and unemployment (Blue)

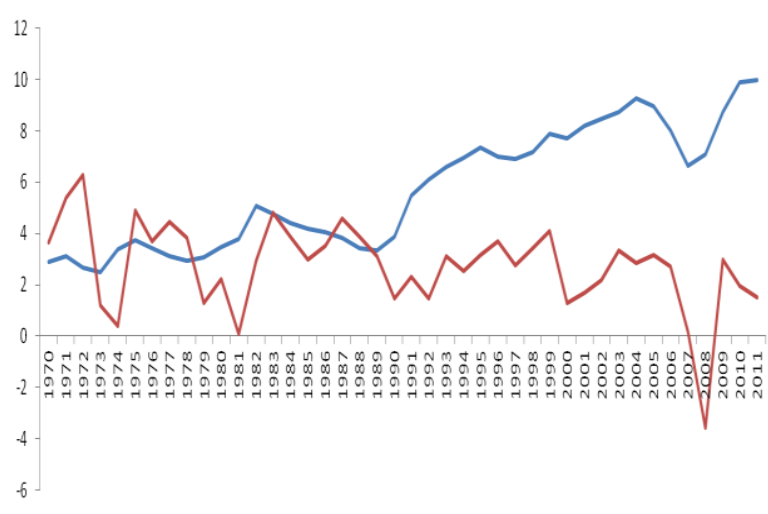

It is seen that; unemployment rates show a continuous trend to increase. Difference between the two variables is $5.586-2.747$ (2.839\%) in the period 1970 to 2012; 7.242-2.303 (4.939\%) in the period 1989 to 2012 and $8.430-1.8716 .559 \%$ in the period 2000 to 2012 . Total GDP for OECD was 3.1 trillion US Dollars in 1970; it has reached 46.1 trillion US Dollars at 2012. This shows that GDP increased 14.87 times.

Technically, Okun's Law suggests that, 3\% increase in GDP causes a 1\% decrease in unemployment (Caraiani, 2010; Elshamy, 2013). Relation between unemployment and growth states the positive effects of power of trade associations and labor costs, when unemployment rates are low and growth rates are high. Besides, efficient trade union bargains in labor market may create negative effects on growth rate (Adjemian, Langor and Rojas 2010). In this study, unemployment and growth data of 23 OECD countries are used. Firstly, Okun Difference Equation (ODE) is used to calculate needed GDP increase to have constant unemployment rate. Then, with the use of time series analysis, long term relation of the two variables is studied.

\section{Literature Review}

First of all, for the different periods and methods, data sets may give different results. For example, while it is observed by Bankole and Fatai (2013) that hypothesis is invalid for Nigeria; Amossoma and Nwosa (2013) found that it is valid. Some studies had gathered results contrasting Okun's Law. For instance, Ting and Ling for Malaysia and Habees and Rumman (2012) for Arabian countries and Jordan showed that, there is no absolute relation between unemployment and growth. Lal and others (2010), for some developing Asian countries, showed that Okun's Law is not applicable. Tillmann (2010) stated that the relation started to get weak from 90 's.

There exist studies showing partial validity for Okun's Law. Some findings are as follows: The relation is unstable for USA and Canada (Beaton, 2010); partially valid for Germany (Oberst and Oelgemöller, 2013); valid with low rate for Central and East Europe (CEE) countries (Hutengs and Stadtmann, 2013); strongly valid for young population and weak validity for old population in Euro zone (Hutengs and Stadtmann, 2012). Also, there are different coefficients for different countries and these coefficients vary in time, while the relation is valid in the opposite way for Euro zone countries (Zanin and Marra, 2012). Using the Italy example, Busetta and Corco (2012) found results suggesting that there might be regional differences. In another study on regional differences, Kangasharju and others (2012) found similar results and pointed the decrease tendency of coefficients. In their study on 
relation of unemployment in crisis periods and growth, for USA and EU, Cazes, Verick and Hussami (2011), and Gordon (2010a; 2010b; 2011) found that after global crisis, coefficients for USA, Canada and Spain showed sudden increases. In addition to this, these increases are quite sudden compared to crisis before 2000 and coefficients are lower for economies with high labor protection, like Germany.

However, many studies provide empirical evidences strongly showing the relation Okun predicts between unemployment and growth. For example; Ball, Leigh and Loungani (2013) for USA and Moazzami and Dadgostar (2009) for 13 OECD countries (for OECD other study: Lee, 2000; Hopkin and Blyth 2012) found that in order to decrease unemployment $1 \%$, increase in GDP should be between $2.6 \%$ - $4.7 \%$. Biggest coefficients in long term are calculated for Canada, Finland, Norway and USA and these countries experienced the effect of economic growth on employment the fastest. In their study for 15 OECD countries, Sögner and Stiassny (2000) found that; there is constant Okun relation for Austria, Belgium, Canada, Italy and USA. There are deviations in Okun's Law for Sweden, Germany, Denmark, Finland, France, Great Britain, Japan, Netherlands, Norway and Switzerland. Herwartz and Niebuhr (2011) showed Okun relation for EU countries, whereas supporting the cause of the differences among the countries with the structural characteristics. Besides, Huang and Lin (2008) for USA and Villaverde and Maza (2008) for Spain found empirical evidence for strong relations. Similar results are found in other country studies (Bakas and Papapetrou, 2012; Ibragimov, Karimov and Permyakova, 2013; Giha, Leat and Renwick, 2012; Mosikari, 2013; Tingii and Lingii, 2011).

Finally, Boulton (2010), for 10 Western Europe countries, showed that $4 \%$ increase in growth causes $1 \%$ decrease in unemployment. Concerning Romania, Andrei, Vasile and Adrian (2009) found that, 0.5\% decrease in growth causes $1 \%$ increase in unemployment. In an analysis on developed countries, Kitov (2011); and Kitov and Kitov, (2012) calculated that the lowest coefficient as 0.4 belonging to Australia and highest as 0.84 belonging to USA. In their study, Huang and Yeh (2013) found that GDP and unemployment variables are co-integrated in long term. Also, they found that these two variables are reversely and strongly related in both long and short term. In a survey study (for Wall Street economists, Mitchell and Pearce, 2009) on G7 countries with professional economists, Pierdzioch, Rülke and Stadtmann (2011) showed that reverse relation between growth and unemployment predicted by economists is parallel to Okun's Law.

\section{Data and Methodology}

The data belonging the period between 1987 and 2012 was taken from the OECD database. An analysis was done for 23 OECD countries' data and total OECD data. First thing in the analysis was to calculate "Okun Coefficient" of countries with the regression relation Okun predicted. Growth rates were categorized as "low" (between 0\%-2\%), "normal" (between 2\%-4\%) and "high" $(4 \%+)$.

In the second step of analysis, the data time series and co-integration relation were tested. For this reason, firstly for each country; i) the growth and unemployment data were tested with unit root tests (Dickey and Fuller. 1979) ii) The two stepped EngleGranger (1987) test was applied. Same process was done on sum of growth and unemployment data of 34 OECD countries. First condition, to decide whether series are co-integrated in long term or not, were to have stationary series at same level.

\section{Empirical Results}

Table 1 shows the results of equation 1 for each country. Regression constants are given in $b_{0}$. $b_{1}$ GDP gives negatively expected coefficient of GDP variable in regressions, known as Okun coefficient. "Average unemployment" (Avg. UNE) and "Average Growth" (Avg. GRW) rates, calculated from the data set of the period 1987 to 2012, are also given on Table 1 . According to this, the highest average unemployment rate is $15.46 \%$, which belongs to Spain and the lowest average unemployment rate is $3.25 \%$, which belongs to Luxembourg. The highest average growth rate is for South Korea with 5.91\%, and the lowest is for Italy with $1.3 \%$.

According to Table 1, average unemployment rate for OECD countries is $6.87 \%$. Mean of average growth rates for the countries with higher average unemployment than this mean (UK, Turkey, Spain, Portugal, Italy, Ireland, Germany, France, Finland, Chile, Canada, Belgium, and Australia) is $3.5 \%$. So, the ratio between average unemployment and average growth is almost two $(0.687 / 0.350=1.96)$. So it can be said that, this reverse relation of unemployment and GDP is oneto-one for the countries with highest unemployment rate.

Mean of average unemployment rates for the countries with lower average unemployment than OECD mean (US, Sweden, Norway, New Zealand, Netherlands, Mexico, Luxembourg, South Korea, Japan and Denmark) is 4.80. Average of average growth rate for these 
countries is 2.76. Even though, this ratio is lower than OECD average, it is pretty close. Ratio between unemployment and GDP is lower $(0.480 / 0.276=1.74)$ for economies with low unemployment rate. Then, findings suggest that average of growth rate is lower $(2.76<3.50)$ for countries with lower unemployment rate than OECD average. Shortly, growth rate occurring when the unemployment is high is higher than growth rate occurring when the unemployment is low. High unemployment creates high growth; low unemployment creates low growth. This is the relation Okun claims.

Table 1: $\quad$ Okun' regressions: $\mathrm{u}-\mathrm{u}_{-1}=\mathrm{b}_{\mathrm{o}}+\mathrm{b}_{1}(\mathrm{y})+\mathrm{e}$ (difference model)

\begin{tabular}{|c|c|c|c|c|c|}
\hline Country & $b_{0}$ & $b_{1}$ (GDP) & $\begin{array}{l}\text { Avg. } \\
\text { UNE }\end{array}$ & $\begin{array}{l}\text { Avg. } \\
\text { GRW }\end{array}$ & $b_{0} / b_{1}$ \\
\hline Australia & 1,139776 & $\begin{array}{c}- \\
0,391112\end{array}$ & 6.919 & 3.301 & 2,91419 \\
\hline Belgium & 0,495702 & $\begin{array}{c}- \\
0,295806\end{array}$ & 8.096 & 1.986 & 1,67576 \\
\hline Canada & 0,841631 & $\begin{array}{c}- \\
0,375152\end{array}$ & 8.173 & 2.484 & 2,24343 \\
\hline Chile & 1,379607 & 0,275437 & 8.23 & 5.698 & 5,00879 \\
\hline Denmark & 0,605353 & $\begin{array}{c}- \\
0,360756\end{array}$ & 5.988 & 1.358 & 1,67801 \\
\hline Finland & 0,879881 & $\begin{array}{c}- \\
0,355040\end{array}$ & 9.13 & 2.138 & 2,47825 \\
\hline France & 0,583921 & $\begin{array}{c}- \\
0,307495 \\
\end{array}$ & 9.423 & 1.805 & 1,89896 \\
\hline Germany & 0,284049 & $\begin{array}{c}- \\
0,151278\end{array}$ & 7.857 & 1.833 & 1,87766 \\
\hline Ireland & 1,595931 & $\begin{array}{c}- \\
0,342655\end{array}$ & 10.2 & 4.871 & 4,65754 \\
\hline Italy & 0,281676 & $\begin{array}{c}- \\
0,216006\end{array}$ & 9.153 & 1.18 & 1,30401 \\
\hline Japan & 0,220478 & $\begin{array}{c}- \\
0,103351\end{array}$ & 3.803 & 1.651 & 2,13329 \\
\hline Korea & 1,118544 & $\begin{array}{c}- \\
0,192760\end{array}$ & 3.369 & 5.911 & 5,80278 \\
\hline $\begin{array}{l}\text { Luxembou } \\
\text { rg }\end{array}$ & 0,328280 & $\begin{array}{c}- \\
0,054827\end{array}$ & 3.257 & 4.085 & 5,98756 \\
\hline Mexico & 0,644013 & $\begin{array}{c}- \\
0,208315\end{array}$ & 3.78 & 2.84 & 3,09153 \\
\hline $\begin{array}{l}\text { Netherland } \\
\mathrm{s}\end{array}$ & 0,487941 & $\begin{array}{c}- \\
0,231328\end{array}$ & 4.769 & 2.268 & 2,10930 \\
\hline $\begin{array}{c}\text { New } \\
\text { Zealand }\end{array}$ & 1,050341 & $\begin{array}{c}- \\
0,374662 \\
\end{array}$ & 6.407 & 2.4 & 2,80343 \\
\hline Norway & 0,237443 & $\begin{array}{c}- \\
0,129464\end{array}$ & 4.134 & 2.337 & 1,83404 \\
\hline Portugal & 1,034295 & $\begin{array}{c}- \\
0,341197\end{array}$ & 6.969 & 2.179 & 3,03137 \\
\hline Spain & 2,512723 & $\begin{array}{c}- \\
0,921603\end{array}$ & 15.469 & 2.559 & 2,72647 \\
\hline Sweden & 0,954893 & $\begin{array}{c}- \\
0,340653\end{array}$ & 6.488 & 2.169 & 2,80312 \\
\hline Turkey & 0,638043 & $\begin{array}{c}- \\
0,157970\end{array}$ & 8.526 & 4.224 & 4,03901 \\
\hline $\begin{array}{c}\text { United } \\
\text { Kingdom }\end{array}$ & 0,564312 & $\begin{array}{c}- \\
0,290173\end{array}$ & 7.061 & 2.363 & 1,94474 \\
\hline $\begin{array}{l}\text { United } \\
\text { States }\end{array}$ & 1,255776 & $\begin{array}{c}- \\
0,449484\end{array}$ & 6.026 & 2.656 & 2,79381 \\
\hline $\begin{array}{c}\text { OECD - } \\
\text { Total }\end{array}$ & 0,716469 & $\begin{array}{c}- \\
0,274117\end{array}$ & 6.873 & 2.438 & 2,61373 \\
\hline
\end{tabular}

Calculated $b_{0} / b_{1}$ coefficients are the "Okun coefficients" and they indicate the needed economic growth rate to prevent unemployment rate. Countries with highest unemployment, Chile (8.23), Ireland (10.2) and Turkey (4.03), need growth rates higher than $4 \%$ to prevent unemployment rate to increase more than current rate. Countries with high calculated Okun coefficient, South Korea (5.80) and Luxembourg (5.98) need growth rate more than $5 \%$.

Lowest Okun coefficient is calculated for these countries: Italy (1.30), Belgium (1.67), Denmark (1.67), Norway (1.83), Germany (1.87), France (1.89) and UK (1.94). These countries have two basic common points: i) Average unemployment is higher than OECD average (except Denmark and Norway) and ii) Average growth rate is lower than OECD average.

The second part of our analysis is time series analysis and to do this, firstly, Dickey-Fuller unit root test is applied to unemployment and growth data of each country. Findings are given in Table 2 . "ADF" column of Table 2 shows ADF statistics of variables for "level" values. Values in parenthesis show the critical value for $5 \%$ meaning value of applied test. For $\mathrm{ADF}<\mathrm{Critical}$ Value, it is concluded that series is not stationary, meaning it includes unit root. In this case, ADF test is redone by taking the first difference of series. ADF test statistics and critical values are calculated taking first difference and given in $\mathrm{ADF}(-1)$.

Unemployment variable for all 23 countries includes unit root in its meaning level. For Italy and Portugal, unemployment variable becomes stationary [I(2)] by taking second difference. Unemployment variable for other 21 countries becomes stationary [I(1)] by taking first difference. For Denmark, Finland, Germany, Italy, Japan, Mexico, Sweden and Turkey, growth variable is stationary in its level value [I(0)]. Growth variable for other 15 countries is stationary [I(1)] when the first difference is taken.

In this case, since for Italy and Portugal unemployment variable is I (2) and for Denmark, Finland, Germany, Italy, Japan, Mexico, Sweden and Turkey growth rate is I (0), they will not be subjected to co-integration analysis. Unemployment and growth relation for other 14 countries is suitable for cointegration analysis. 
Table 2: $\quad$ ADF Unit root tests for UNE and GRW

\begin{tabular}{|c|c|c|c|c|c|c|c|c|c|c|c|}
\hline \multirow[b]{2}{*}{ Country } & \multicolumn{2}{|c|}{ UNE } & \multicolumn{2}{|c|}{ GRW } & \multirow{2}{*}{ Notes } & & & & & & \multirow{4}{*}{$\begin{array}{l}\mathrm{I}(0) \text {; } \\
\text { GRW } \\
\text { is } \\
\text { Meani } \\
\text { ngful } \\
\text { at I }(0) \\
\text { for } 1 \%\end{array}$} \\
\hline & $\mathrm{ADF}$ & $\operatorname{ADF}(-1)$ & $\mathrm{ADF}$ & $\operatorname{ADF}(-1)$ & & & & & & & \\
\hline Australia & $\begin{array}{c}-0,908449 \\
(-1,955020)\end{array}$ & $\begin{array}{c}-3,248560 \\
(-1,955681)\end{array}$ & $\begin{array}{c}-1,538109 \\
(-1,955020)\end{array}$ & $\begin{array}{c}-5,681636 \\
(-1,955681)\end{array}$ & & & & & & & \\
\hline \multirow{3}{*}{ Belgium } & \multirow[b]{3}{*}{$\begin{array}{c}0,142256 \\
(-1,958088) \\
\end{array}$} & \multirow[b]{3}{*}{$\begin{array}{c}-4,130355 \\
(-1,958088) \\
\end{array}$} & \multirow[b]{3}{*}{$\begin{array}{c}-2,321835 \\
(-2,660720)^{*}\end{array}$} & \multirow[b]{3}{*}{$\begin{array}{c}-5,797556 \\
(-1,956406) \\
\end{array}$} & \multirow{3}{*}{$\begin{array}{l}\text { GRW } \\
\text { is } \\
\text { Meani } \\
\text { ngful } \\
\text { at } \mathrm{I}(0) \\
\text { for } 1 \% \\
\end{array}$} & & & & & & \\
\hline & & & & & & Spain & $\begin{array}{c}0,174815 \\
(-1,955681)\end{array}$ & $\begin{array}{c}-1,955758 \\
(-1,955681)\end{array}$ & $\begin{array}{c}-1,767823 \\
(-1,955020)\end{array}$ & $\begin{array}{c}-4,735233 \\
(-1,955681)\end{array}$ & \\
\hline & & & & & & \multirow[b]{2}{*}{ Sweden } & & & & & $\begin{array}{l}\text { GRW } \\
\text { is }\end{array}$ \\
\hline \multirow{2}{*}{ Canada } & & & & & $\begin{array}{l}\text { GRW } \\
\text { is } \\
\text { Meani }\end{array}$ & & $\begin{array}{c}-0,291357 \\
(-1,955681)\end{array}$ & $\begin{array}{c}-3,413449 \\
(-1,956408)\end{array}$ & $\begin{array}{c}-2,828183 \\
(-1,955020)\end{array}$ & & $\begin{array}{l}\text { Station } \\
\text { ary at } \\
\mathrm{I}(0)\end{array}$ \\
\hline & $\begin{array}{c}-0,611939 \\
(-1,955020) \\
\end{array}$ & $\begin{array}{c}-3,474439 \\
(-1,955681) \\
\end{array}$ & $\begin{array}{c}-2,005565 \\
(-2,660720)^{*}\end{array}$ & $\begin{array}{c}-5,675185 \\
(-1,955681) \\
\end{array}$ & $\begin{array}{l}\text { ngful } \\
\text { at I(0) } \\
\text { for } 1 \%\end{array}$ & \multirow[t]{2}{*}{ Turkey } & & & & & $\begin{array}{l}\text { GRW } \\
\text { is } \\
\text { Station }\end{array}$ \\
\hline Chile & $\begin{array}{c}-1,134512 \\
(-1,955020) \\
\end{array}$ & $\begin{array}{c}-4,425504 \\
(-1,955681) \\
\end{array}$ & $\begin{array}{c}-1,447020 \\
(-1,955020) \\
\end{array}$ & $\begin{array}{c}-6,924916 \\
(-1,955681) \\
\end{array}$ & & & $\begin{array}{c}-0,301171 \\
(-1,955020)\end{array}$ & $\begin{array}{c}-4,336725 \\
(-1,955681)\end{array}$ & $\begin{array}{c}-3,716088 \\
(-1,955020)\end{array}$ & & $\begin{array}{l}\text { ary at } \\
\mathrm{I}(0)\end{array}$ \\
\hline \multirow{4}{*}{ Denmark } & \multirow{4}{*}{$\begin{array}{c}0,169550 \\
(-1,955020) \\
\end{array}$} & \multirow{4}{*}{$\begin{array}{c}-3,404381 \\
(-1,955681) \\
\end{array}$} & \multirow{4}{*}{$\begin{array}{c}-2,720146 \\
(-1,955020) \\
\end{array}$} & & \multirow{4}{*}{\begin{tabular}{|l} 
GRW \\
is \\
Station \\
ary at \\
I( 0$)$ \\
GRW
\end{tabular}} & $\begin{array}{c}\text { United } \\
\text { Kingdom }\end{array}$ & $\begin{array}{c}-0,270641 \\
(-1,955681) \\
\end{array}$ & $\begin{array}{c}-3,224373 \\
(-1,955681) \\
\end{array}$ & $\begin{array}{c}-2,119882 \\
(-1,955020) \\
\end{array}$ & $\begin{array}{c}-5,024712 \\
(-1,956406) \\
\end{array}$ & \\
\hline & & & & & & $\begin{array}{l}\text { United } \\
\text { States }\end{array}$ & $\begin{array}{c}0,275519 \\
(-1,956406)\end{array}$ & $\begin{array}{c}-3,844323 \\
(-1,956406)\end{array}$ & $\begin{array}{c}-1,541823 \\
(-1,955020)\end{array}$ & $\begin{array}{c}-6,018883 \\
(-1,955681)\end{array}$ & \\
\hline & & & & & & \multirow{2}{*}{$\begin{array}{c}\text { OECD - } \\
\text { Total }\end{array}$} & \multirow{2}{*}{$\begin{array}{c}0,616892 \\
(-1,956406)\end{array}$} & \multirow{2}{*}{$\begin{array}{c}-4,191554 \\
(-1,956406)\end{array}$} & \multirow{2}{*}{$\begin{array}{c}-1,859498 \\
(-1,955020)\end{array}$} & \multirow{2}{*}{$\begin{array}{c}-6,710746 \\
(-1,955681)\end{array}$} & \\
\hline & & & & & & & & & & & \\
\hline
\end{tabular}

Results of applied test for 14 countries having stationary variables at the same level, which is prerequisite for Engle-Granger co-integration test, and all of OECD are given on Table 3.

Engle-Granger co-integration test examines the long term integration relation between variables. For this purpose, firstly, regression models between unemployment (independent) and growth (dependent) for each country are designed. Calculated coefficients, standard error and t-statistics for regression models are given on Table 3 . Being stationary in their level of $U$ model, error term is prerequisite for two variables to be co-integrated in long term. ADF unit-root test is applied to create error term series and calculated ADF test statistics are given in "u ADF" and critical value for 5\% meaning level of these values. They are presented in " $5 \%$ level" column. When these two columns are examined, it is seen that error terms are stationary $[\mathrm{I}(0)]$ at their level value. In this case, it can be concluded that unemployment and growth variables are co-integrated in long term for each country.

Table 3: $\quad$ Engle-Granger cointegration test

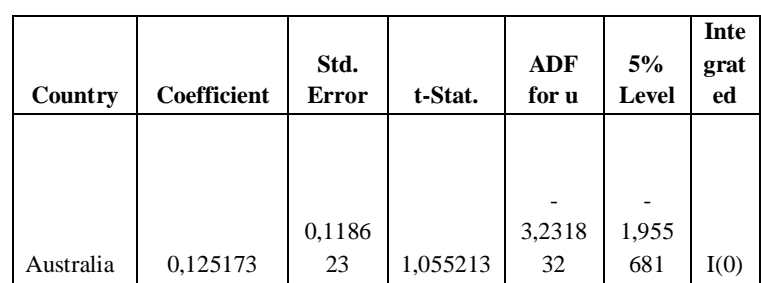

Okun's Law and Long Term Co-Integration Analysis

for OECD Countries (1987-2012) 


\begin{tabular}{|c|c|c|c|c|c|c|}
\hline Belgium & $-0,006537$ & $\begin{array}{c}0,0779 \\
58\end{array}$ & $\begin{array}{c}- \\
0,083850\end{array}$ & $\begin{array}{c}- \\
3,8133 \\
56\end{array}$ & $\begin{array}{c}- \\
1,958 \\
088\end{array}$ & $\mathrm{I}(0)$ \\
\hline Canada & $-0,140804$ & $\begin{array}{c}0,0737 \\
00\end{array}$ & $\begin{array}{c}- \\
1,910512\end{array}$ & $\begin{array}{c}4,0585 \\
20\end{array}$ & $\begin{array}{c}1,956 \\
406\end{array}$ & $\mathrm{I}(0)$ \\
\hline Chile & $-0,157108$ & $\begin{array}{c}0,0621 \\
89\end{array}$ & $\begin{array}{c}- \\
2,526298\end{array}$ & $\begin{array}{c}- \\
4,2176 \\
02\end{array}$ & $\begin{array}{c}- \\
1,955 \\
681\end{array}$ & $\mathrm{I}(0)$ \\
\hline France & $-0,068150$ & $\begin{array}{c}0,0794 \\
65\end{array}$ & $\begin{array}{c}- \\
0,857608\end{array}$ & $\begin{array}{c}- \\
3,3065 \\
43\end{array}$ & $\begin{array}{c}- \\
1,955 \\
671\end{array}$ & $\mathrm{I}(0)$ \\
\hline Ireland & $-0,195960$ & $\begin{array}{c}0,1009 \\
40\end{array}$ & $\begin{array}{c}- \\
1,941340\end{array}$ & $\begin{array}{c}- \\
2,3351 \\
31\end{array}$ & $\begin{array}{c}- \\
1,955 \\
681\end{array}$ & $\mathrm{I}(0)$ \\
\hline Korea & $-0,097530$ & $\begin{array}{c}0,0397 \\
06\end{array}$ & $\begin{array}{c}- \\
2,456297\end{array}$ & $\begin{array}{c}- \\
4,9980 \\
89\end{array}$ & $\begin{array}{c}- \\
1,956 \\
406\end{array}$ & $\mathrm{I}(0)$ \\
\hline $\begin{array}{c}\text { Luxembo } \\
\text { urg }\end{array}$ & $-0,037914$ & $\begin{array}{c}0,0255 \\
35\end{array}$ & $\begin{array}{c}- \\
1,484799\end{array}$ & $\begin{array}{c}- \\
3,5224 \\
90\end{array}$ & $\begin{array}{c}- \\
1,955 \\
681\end{array}$ & $\mathrm{I}(0)$ \\
\hline $\begin{array}{c}\text { Netherlan } \\
\text { ds }\end{array}$ & 0,006223 & $\begin{array}{c}0,0752 \\
30\end{array}$ & 0,082718 & $\begin{array}{c}- \\
3,7949 \\
21\end{array}$ & $\begin{array}{c}- \\
1,957 \\
204\end{array}$ & $\mathrm{I}(0)$ \\
\hline $\begin{array}{c}\text { New } \\
\text { Zealand }\end{array}$ & $-0,025766$ & $\begin{array}{c}0,0947 \\
66\end{array}$ & $\begin{array}{c}- \\
0,271893\end{array}$ & $\begin{array}{c}- \\
2,9139 \\
87\end{array}$ & $\begin{array}{c}- \\
1,955 \\
681\end{array}$ & $\mathrm{I}(0)$ \\
\hline Norway & 0,009446 & $\begin{array}{c}0,0658 \\
29\end{array}$ & 0,143499 & $\begin{array}{c}- \\
3,6410 \\
56\end{array}$ & $\begin{array}{c}- \\
1,956 \\
406\end{array}$ & $\mathrm{I}(0)$ \\
\hline Spain & $-0,625511$ & $\begin{array}{c}0,2378 \\
83\end{array}$ & $\begin{array}{c}- \\
2,629487\end{array}$ & $\begin{array}{c}- \\
1,9865 \\
34\end{array}$ & $\begin{array}{c}- \\
1,955 \\
681\end{array}$ & $\mathrm{I}(0)$ \\
\hline $\begin{array}{c}\text { United } \\
\text { Kingdom }\end{array}$ & $-0,046496$ & $\begin{array}{c}0,0776 \\
38\end{array}$ & $\begin{array}{c}- \\
0,598886\end{array}$ & $\begin{array}{c}- \\
3,0048 \\
77\end{array}$ & $\begin{array}{c}- \\
1,955 \\
681\end{array}$ & $\mathrm{I}(0)$ \\
\hline $\begin{array}{l}\text { United } \\
\text { States }\end{array}$ & $-0,150388$ & $\begin{array}{c}0,1025 \\
29\end{array}$ & $\begin{array}{c}- \\
1,466780\end{array}$ & $\begin{array}{c}- \\
4,2560 \\
37\end{array}$ & $\begin{array}{c}- \\
1,956 \\
406\end{array}$ & $\mathrm{I}(0)$ \\
\hline $\begin{array}{c}\text { OECD - } \\
\text { Total }\end{array}$ & $-0,092221$ & $\begin{array}{c}0,0540 \\
69\end{array}$ & $\begin{array}{c}- \\
1,705604\end{array}$ & $\begin{array}{c}- \\
4,6535 \\
30\end{array}$ & $\begin{array}{c}- \\
1,956 \\
406\end{array}$ & $\mathrm{I}(0)$ \\
\hline
\end{tabular}

Taking first difference, because series are not stationary while examining the long term co-integration relation, they create short term information losses. Removing these losses, Error Correction Models are degisned to show existence of short term relation and the results are presented on Table 4.

By using, for each country, unemployment (dependent), growth (independent) variables and 1 lagged error term series in Error Correction Models, VAR models are obtained and obtained coefficients, error term coefficients (u (-1) coefficients), t statistics and probability values for $5 \%$ meaning level are provided on Table 4.

Tablo 4: $\quad$ Error Correction Models

\begin{tabular}{|c|c|c|c|c|c|}
\hline Country & $\begin{array}{c}\text { Coefficien } \\
\mathbf{t}\end{array}$ & $\begin{array}{c}\mathbf{u} \text { (-1) } \\
\text { Coefficien } \\
\mathbf{t}\end{array}$ & t-Stat. & $\begin{array}{c}\mathbf{5 \%} \\
\text { Prob. }\end{array}$ & Notes \\
\hline & & & & & \\
Australia & $-0,071853$ & $-0,568314$ & 0,226474 & 0,0204 & $\begin{array}{c}56 \% \text { of losses } \\
\text { is removed } \\
\text { after 1 period }\end{array}$ \\
\hline
\end{tabular}

\begin{tabular}{|c|c|c|c|c|c|}
\hline Belgium & $-0,571773$ & $-0,571773$ & 2,637700 & 0,0154 & $\begin{array}{l}57 \% \text { of losses } \\
\text { is removed } \\
\text { after } 1 \text { period }\end{array}$ \\
\hline Canada & $-2,296605$ & $-0,718622$ & 3,137732 & 0,0050 & $\begin{array}{l}71 \% \text { of losses } \\
\text { is removed } \\
\text { after } 1 \text { period }\end{array}$ \\
\hline Chile & $-0,177540$ & 0,164910 & 0,682898 & 0,5021 & $\begin{array}{c}\text { Losses con } \\
\text { not be } \\
\text { removed }\end{array}$ \\
\hline France & $-0,246964$ & $-0,680557$ & 2,465309 & 0,0224 & $\begin{array}{l}68 \% \text { of losses } \\
\text { is removed } \\
\text { after } 1 \text { period }\end{array}$ \\
\hline Ireland & $-0,280638$ & $-0,662728$ & 0,174254 & 0,0010 & $\begin{array}{l}66 \% \text { of losses } \\
\text { is removed } \\
\text { after } 1 \text { period }\end{array}$ \\
\hline Korea & $-0,131000$ & 0,315185 & 1,236727 & 0,2298 & $\begin{array}{c}\text { Losses con } \\
\text { not be } \\
\text { removed }\end{array}$ \\
\hline $\begin{array}{l}\text { Luxembou } \\
\text { rg }\end{array}$ & $-0,032472$ & 0,315044 & 1,552124 & 0,1356 & $\begin{array}{l}\text { Losses con } \\
\text { not be } \\
\text { removed }\end{array}$ \\
\hline $\begin{array}{c}\text { Netherland } \\
\text { s }\end{array}$ & $-0,090831$ & $-0,698149$ & 3,642073 & 0,0015 & $\begin{array}{l}69 \% \text { of losses } \\
\text { is removed } \\
\text { after } 1 \text { period }\end{array}$ \\
\hline $\begin{array}{c}\text { New } \\
\text { Zealand }\end{array}$ & $-0,093035$ & $-0,526498$ & 2,955737 & 0,0075 & $\begin{array}{l}52 \% \text { of losses } \\
\text { is removed } \\
\text { after } 1 \text { period }\end{array}$ \\
\hline Norway & $-0,066788$ & $-0,553571$ & 2,629159 & 0,0157 & $\begin{array}{l}55 \% \text { of losses } \\
\text { is removed } \\
\text { after } 1 \text { period }\end{array}$ \\
\hline Spain & $-0,861976$ & $-0,750691$ & 4,553393 & 0,0002 & $\begin{array}{l}75 \% \text { of losses } \\
\text { is removed } \\
\text { after } 1 \text { period }\end{array}$ \\
\hline $\begin{array}{l}\text { United } \\
\text { Kingdom }\end{array}$ & $-0,224679$ & $-0,823409$ & 4,693064 & 0,0001 & $\begin{array}{l}82 \% \text { of losses } \\
\text { is removed } \\
\text { after } 1 \text { period }\end{array}$ \\
\hline $\begin{array}{l}\text { United } \\
\text { States }\end{array}$ & $-0,277950$ & $-0,564665$ & 2,567682 & 0,0179 & $\begin{array}{l}56 \% \text { of losses } \\
\text { is removed } \\
\text { after } 1 \text { period }\end{array}$ \\
\hline $\begin{array}{c}\text { OECD - } \\
\text { Total }\end{array}$ & $-0,186823$ & $-0,600909$ & 2,514537 & 0,0201 & $\begin{array}{c}60 \% \text { of losses } \\
\text { is removed } \\
\text { after } 1 \text { period }\end{array}$ \\
\hline
\end{tabular}

According to Table 4, Error Correction Model does not work for Chile, South Korea and Luxembourg. Model works for other 12 countries and by removing data losses caused by long term, short term relation is showed. According to this, for these countries, a ratio is given in "Notes" column, and data loss for any term can be removed in the following period.

\section{Results}

Okun Law indicates reverse relation between unemployment and growth. This means that, increase in unemployment causes decrease in GDP. Results of calculation related to OECD countries showed that Okun Law is valid for 23 countries. Growth performance of countries, especially with high employment rate, is quite low. Countries, especially with high Okun coefficient, need that economic growth to keep unemployment at current rate.

Long term co-integration is valid for 14 of 23 OECD countries and variables are long term related. 
Similar results of Huang and Yeh (2013) for long term relation are found. Finally, the findings of Tillmann (2010) which state that reverse relation between unemployment and growth in the long term get weak is supported. For 34 OECD countries, average unemployment is calculated as 6.87 and average growth is computed as 2.43. These results show that, long term relation of unemployment and growth data are suggesting validity for Okun's Law.

\section{REFERENCES}

Adjemian, S., Langor, F. \& Rojas, C. Q. (2010). How do labor market institutions affect the link between growth and unemployment: The case of the European countries. The Eeuropean Journal of Comparative Economics, 7(2), 347-371.

Amassoma, D. \& Nwosa, P. I. (2013). The impact of unemployment rate on productivity growth in Nigeria: A error correction modeling approach. International Journal of Economics and Management Sciences, 2(8), 01-13.

Andrei, D. B., Vasile, D. \& Adrian, E. (2009). The correlation between unemployment and real GDP growth. A study case on Romania. Annals of Faculty of Economics, 2(1), 317-322.

Bakas, D. \& Papapetrou, E. (2012).Unemployment in Greece: Evidence from Greek regions. Bank of Greece, Working Paper, No.146.

Ball, L., Leigh, D. \& Loungani, P. (2013). Okun's Law: Fit at 50? NBER Working Paper, No.18668.

Bankole, A. S. \& Fatai, B. O. (2013). Empirical test of Okun's Law in Nigeria. International Journal of Economic Practices and Theories, 3(3), 227-231.

Beaton, K. (2010). Time variation in Okun's Law: A Canada and U.S. comparison. Bank of Canada Working Paper, No.7.

Boulton, T. (2010). Test of Okun's Law fort the 10 Eastern Europen countries. London Metropolitan Business School, Economics Subject Group.
Busetta, G. \& Corso, D. (2012) Labor productivity and Okun's Law: An emprical application on Italian regional panel data. Rivista Italiana Degli Economesti, 2, 279-298.

Caraiani, P. (2010). Bayesian linear estimation of Okun coefficient for Romania: Sensitivity to priors distributions. The Romanian Economic Journal, 38, 5365.

Cazes, S., Verick, S. \& Hussami, F. A. (2011). Diverging trends in unemployment in the United States and Europe: Evidence from Okun's Law and the global financial crisis. International Labor Office, Employment Working Paper, No.106.

Dickey, D. A., \& Fuller, W. A. (1979). Distribution of the estimators of autoregressive time series with a unit root. Journal of the American Statistical Association, 74, 427-431.

Elshamy, H. (2013). The relationship between unemployment and output in Egypt. Procedia Social and Behavioral Sciences, 81, 22-26.

Engle, R. F., \& Granger, C. W. J. (1987). "Cointegration and error correction: Representation, estimation, and testing. Econometrica, 55(2), 251-276.

Giha, C. R., Leat, P. M. \& Renwick, A. W. (2012). The relationship between output and employment in Scotland: A regional analysis. Scotland's Rural College, Working Papers, No.131465.

Gordon, R. J. (2010a). Revisiting and rethinking the business cycle: Okun's Law and productivity innovations. American Economic Review, 100, 1115.

Gordon, R. J. (2010b). The demise of Okun's Law and of procyclical fluctuations in conventional and unconventional measures of productivity. For Presentation at NBER Summer Institute, PRBB Research Meeting, Cambridge.

Gordon, R. J. (2011). The evolution of Okun's Law and of cyclical productivity 
fluctuations in the United States and in the EU-15. For Presentation at EES/IAB Workshop, Nuremberg.

Habees, M. A. \& Rumman, M. A. (2012). The relationship between unemployment and economic growth in Jordan and some Arab countries. World Applied Sciences Journal. 18(5), 673-680.

Herwartz, H. \& Niebuhr, A. (2011). Growth, unemployment and labor market institutions: Evidence from a crosssection of EU regions, Applied Economics, 43(30), 4663-4676.

Hopkin, J. \& Blyth, M. (2012). What can Okun teach Polanyi? Efficiency, regulation and equality in the OECD. Review of International Political Economy, 19(1), $1-33$

Huang, H. C. \& Lin, S. C. (2008). Smoothtime-varying Okun's coefficients. Economic Modelling, 25, 363-375.

Huang, H. C. \& Yeh, C. C. (2013). Okun's Law in panels of countries and state. Applied Economics, 45(2), 191-199.

Hutengs, O. \& Stadtmann, G. (2012). Age effects in the Okun's Law within the Eurozone. German Institute for Economic Research, Discussion Papers, No.1243.

Hutengs, O. \& Stadtmann, G. (2013). Don't trust anybody over 30: Youth unemployment and Okun's Law in CEE countries. European University Viadrina Frankfurt, Department of Business Administration and Economics, Discussion Paper, No.333.

Ibragimov, M., Karimov, J. \& Permyakova, E. (2013). Unemployment and output Dynamics in CIS countries: Okun's law revisited. EERC, Working Paper Series, No.13/04.

Kangasharju, A., Tavera, C. \& Nijkamp, P. (2012). Regional growth and unemployment: The validity of Okun's Law for the finnish regions, Spatial Economic Analysis, 7(3), 381-395.

Kitov, I. O. (2011). Okun's Law revisited. Is there structural unemployment in developed countries?. MPRA Paper, No.32135.
Kitov, I. \& Kitov, O., (2012). Modeling unemployment and employment in advanced economies: Okun's Law with a structural break. Theoretical and Practical Research in Economic Fields, 5(3), 26-41.

Knotek, E. S. (2007). "How useful is Okun's Law?. Federal Reserve Bank of Kansas, Journal Economic Review, 4, 73-103.

Lal, I., Muhammad, S. D., Jalil, M. A. \& Hussain, A. (2010). Test of Okun's Law in some Asian countries cointegration approach. European Journal of Scientific Research, 40(1), 73-80.

Lang, D. \& De Peretti, C. (2009). A strong hysteretic model for Okun's Law: Theory and preliminary investigation. International Review of Applied Economics, 23(4), 445-462.

Lee, J. (2000). The robustness of Okun's Law: Evidence from OECD countries. Journal of Macroeconomics, 22(2), 331-356.

Levine, L., (2013). Economic growth and the unemployment rate. CRS Report for Congress.

Mankiw, (2010). Macroeconomics. Seventh Edition, Worth Publishers, New York.

Mitchell, K. \& Pearce, D. K. (2009). Do Wall Street economicsts believe in Okun's Law and the Taylor rule? Journal of Economics and Finance, 34(2), 196217.

Moazzami, B. \& Dadgostar, B. (2009). Okun's Law revisited: Evidence from OECD Countries. International Business and Economics Research Journal. 8(8), 2124.

Mosikari, T. J. (2013). The effect of unemployment rate on gross domestic product: Case of South Africa. Mediterranean Journal of Social Sciences, 4(6), 429-434.

Oberst, C. A. \& Oelgemöller, J. (2013). Economic growth and regional labor market development in German regions: Okun's Law in a spatial context. FNC Working Paper, No:5. 
Okun, A. M. (1962). Potential GNP: Its measurement and significance. Proceedings of the Business and Economics Statistics Section of the American Statistical Association. 98104.

Pierdzioch, C., Rülke, J. C. \& Stadtmann, G. (2011). Do Professional economists' forecasts reflect Okun's Law? Some evidence fort the G7 countries, Applied Economics, 43(11), 1365-1373.

Plosser, C. I. \& Schwert, G. W. (1979). "Potential GNP: Its Measurement and Significance: A Dissenting Opinion", Journal of Monetary Economics, 10, 179-186.

Sögner, L. \& Stiassny, A. (2000). A crossCountry study on Okun's Law. Vienna University of Economics and Business Administration, Working Paper Series, No:13.

Teck, H. H. (2012). An economic analysis of optimum population size achieved through boosting total fertility and net immigration. National University of Singapore, Institute of Policy Studies.

Tillmann, P. (2010). Do FOMC members believe in Okun's Law?. Economics Bulletin, 30(3), 2398-2404.

Tingi, N. Y. \& Lingii, L. S. (2011). Okun's Law in Malaysia: An autoregressive distributed LAG (ARDL) approach with Hodrick-Prescott (HP) fitler. Journal of Global Business and Economics, 2(1), 95-103.

Villaverde, J. \& Maza, A. (2008). The robustness of Okun's Law in Spain: 1980-2001 Regional evidence. Journal of Policy Modeling, 31, 289-297.

Zanin, L. \& Marra, G. (2012). Rolling regression versus time-varying coefficient modelling: An empirical investigation of the Okun's Law in some Euro Area countries. Bulletin of Economic Research, 64(1), 91-108. 